\title{
Elaboração e análise sensorial de biscoito recheado enriquecido com fibras e com menor teor de gordura
}

\author{
Development and sensorial analysis of stuffed cookie enriched with fibers and with \\ lower fat content
}

\author{
Beatriz Mortari Saydelles ${ }^{I}$ Viviani Ruffo de Oliveira' ${ }^{I I}$ Vanessa Bordin VieraI \\ Clândio Timm Marques ${ }^{\mathrm{I}}$ Claudia Severo da Rosa ${ }^{\text {III }}$
}

\begin{abstract}
Atualmente, observa-se que as crianças consomem, em grande quantidade, alimentos contendo excesso de gorduras e pobres em fibras como: balas, salgadinhos, alimentos fast food e biscoitos recheados. Com a necessidade de se elaborar alimentos mais saudáveis, buscou-se por meio do presente estudo elaborar um biscoito recheado enriquecido com fibras e com menor teor de gordura, analisar a composição de fibras e lipídeos e verificar a aceitabilidade do biscoito elaborado em comparação a um biscoito industrializado. As análises de fibra bruta e lipídeos foram realizadas em duplicatas, segundo metodologia descrita pela AOAC (1995). A análise sensorial foi realizada com 50 crianças, com idades entre sete e 10 anos, em uma Instituição Privada de Santa Maria, Rio Grande do Sul (RS), utilizando uma ficha de avaliação com escala hedônica facial de três pontos. Houve redução no teor de gorduras e aumento no teor de fibras do biscoito elaborado. Além disso, verificou-se que o biscoito elaborado obteve uma boa aceitação, não diferindo estatisticamente do industrializado.
\end{abstract}

Palavras-chave: escolares, fibra alimentar, lipídios.

\section{ABSTRACT}

Nowadays, it is observed that children consume food containing a large amount of fat and low quantity of fiber such as: candies, snacks, fast food and filled cookies. Because of the need to develop healthier food, the aim of this study was to develop cookie enriched with fiber and less fat content, analyze the fiber and fat composition and verify the acceptability of the cookies in comparison to an industrialized cookie. The analysis of crude fiber and fat were performed in duplicates according to AOAC (1995) methodology. The sensory analysis was performed with 50 children aged between 7 and 10 yearsold at a private institution in Santa Maria - RS, using as evaluation form a hedonic facial scale with three points. According to the results it can be concluded that cookie prepared had a good acceptance, without statistics difference between the industrialized one, as well as, achieved a reduction in fat content and an increase in the fiber content.

Key words: scholars, dietary fiber, lipids.

Apesar de todas as evidências de que uma alimentação inadequada propicia o desenvolvimento de vários tipos de doenças, muitas vezes desencadeadas na infância, cada vez mais observa-se que as crianças consomem, em grande quantidade, alimentos contendo excesso de gorduras e pobres em fibra como: balas, salgadinhos, alimentos de fast food e biscoitos recheados (GALLAGHER et al., 2003).

O biscoito é um produto composto principalmente por farinha de trigo, gordura e açúcar, com teor de umidade bastante baixo, o que lhe proporciona uma longa vida de prateleira, principalmente se acondicionado em embalagem eficiente na proteção de umidade (MONTEIRO, 1996).

Esses produtos geralmente são consumidos para satisfazer as necessidades sensoriais, e não nutricionais, sendo assim, a qualidade sensorial é o

ICentro Universitário Franciscano (UNIFRA), Santa Maria, RS, Brasil.

IUniversidade Federal do Rio Grande do Sul (UFRGS), 90040-060, Porto Alegre, RS, Brasil. E-mail: vivianiruffo@hotmail.com. Autor para correspondência.

IIIDepartamento de Tecnologia e Ciência dos Alimentos, Universidade Federal de Santa Maria (UFSM), Santa Maria, RS, Brasil. 
principal fator na determinação da aceitação e da preferência do consumidor por esses produtos, devendo-se conhecer os parâmetros sensoriais considerados importantes por esse público (ORMENESE et al., 2001).

As fibras alimentares desenvolvem papéis importantes no trato gastrintestinal humano. Além de diminuírem a absorção de gorduras, aumentarem o peristaltismo intestinal e produzirem ácidos graxos de cadeia curta, atuantes no combate ao colesterol, também promovem a regulação no tempo de trânsito intestinal e apresentam alto poder de saciedade. Destaca-se que essas propriedades fisiológicas são essenciais para o tratamento e a prevenção das complicações oriundas da obesidade (LIMA et al., 2004; PITEIRA et al., 2006).

Reconhecida como excelente fonte de fibra, as sementes de linhaça contêm fibras como hemicelulose, celulose e lignina, auxiliando a prover uma fonte rica de ácidos graxos ômega-3 na dieta, além de ser rica em minerais como cálcio e fósforo (PUFFET, 2004).

Em razão da necessidade de se desenvolverem alimentos mais saudáveis para que se minimizem erros alimentares na vida adulta, este trabalho buscou elaborar biscoito recheado enriquecido com fibras e com menor teor de gordura, analisar a composição de fibras e lipídeos e verificar a aceitabilidade do biscoito elaborado em comparação a um biscoito industrializado.

A presente pesquisa caracterizou-se como um estudo quantitativo experimental, o qual foi desenvolvido no Laboratório de Técnica Dietética de um Curso de Nutrição.

Na formulação do biscoito, foram utilizados: farinha de trigo (40g), farinha de trigo integral (110g), amido de milho (30g), achocolatado em pó (40g), cacau em pó (10g), linhaça triturada (15g), açúcar (110g), margarina light (30g) e ovo (58g). Todos os ingredientes foram adquiridos em estabelecimentos comerciais da cidade de Santa Maria, Rio Grande do Sul (RS), e foram pesados em balança digital (Filizola).

O processo de elaboração do biscoito iniciou com a homogeneização do ovo e da margarina. Em seguida, foram acrescentados a farinha de trigo, o amido de milho, o achocolatado, o açúcar, o cacau em pó, a linhaça triturada e, por último, a farinha de trigo integral, misturando bem até obter uma massa homogênea. Após repouso por 20 minutos, a massa foi aberta com um rolo e os biscoitos foram cortados com um cortador próprio. Os biscoitos foram dispostos em assadeiras devidamente untadas e levados para assar em forno convencional, sob temperatura de $150^{\circ} \mathrm{C}$, por 10 minutos. Com o auxílio de uma espátula, os biscoitos foram retirados da assadeira e, após esfriarem, distribuiu-se de maneira uniforme o recheio em uma parte do biscoito, cobrindo-a com a outra parte do biscoito.

Foram homogeneizados o leite em pó desnatado (85g), o açúcar (150g) e a água fervente (80ml) em liquidificador de marca Britânia Modelo LQDiamante, com alta velocidade até se obter uma consistência firme. A mistura foi vertida em recipiente, no qual foram adicionados margarina (10g) e achocolatado em pó (20g), deixando cozinhar em fogo baixo, mexendo sempre, até não aderir mais ao recipiente.

As análises de composição de fibra bruta e de lipídeos foram realizadas em duplicadas, no Laboratório de Bromatologia do Centro Universitário Franciscano (UNIFRA), e no Laboratório de Ciência e Tecnologia de Alimentos (CTA) da Universidade Federal de Santa Maria (UFSM), respectivamente, seguindo a metodologia descrita pela Association Of Official Analytical Chemists (AOAC, 1995).

A análise sensorial foi realizada com 50 crianças de uma Instituição Privada do Município de Santa Maria - RS, com idade entre sete e 10 anos. Cada criança recebeu duas amostras de biscoito de sabor chocolate, sendo uma composta pelo biscoito recheado elaborado (BRE) neste estudo, e outra por um biscoito recheado industrializado (BRI) adquirido em um estabelecimento comercial de Santa Maria - RS.

As amostras foram dispostas em pratos brancos codificados com três dígitos aleatórios. Além disso, também foi fornecido um copo com água, para proporcionar a limpeza das papilas gustativas entre uma amostra e outra. A avaliação foi realizada por meio de uma ficha de avaliação contendo uma escala hedônica facial variando de 1 a 3 pontos, na qual ancorava os eixos 1 correspondente a "gostei muito" e o eixo 3 a “desgostei muito”.

Participaram da análise sensorial somente as crianças cujos os responsáveis assinaram o Termo de Consentimento Livre e Esclarecido (TCLE), aprovado pelo Comitê de Ética da UNIFRA, seguindo a Resolução nº196/96.

Os dados obtidos foram analisados por meio de análise de variância e, para a comparação das médias entre as amostras, utilizou-se o teste de T, com um nível de significância de 5\%. Para a análise dos dados, foi utilizado o programa Statistic versão 6.0.

Como pode ser observado na tabela 1 , o biscoito recheado elaborado (BRE) obteve 5,46g de fibras em $100 \mathrm{~g}$ de biscoito, enquanto que o biscoito recheado industrializado (BRI) apresenta 2,66g de fibras em $100 \mathrm{~g}$ do biscoito de acordo com o rótulo. Esses resultados demonstram haver diferença estatística significativa entre o biscoito recheado elaborado e o industrializado, o que se deve provavelmente à adição de farinha de trigo integral associada à linhaça triturada para a formulação elaborada neste estudo. 
Tabela 1 - Médias e desvios-padrão da composição química, em g $100 \mathrm{~g}^{-1}$, do biscoito recheado elaborado (BRE) e do biscoito recheado industrializado (BRI). Santa Maria RS.

\begin{tabular}{ccc}
\hline Componentes avaliados & BRE & \multicolumn{1}{c}{ BRI } \\
\hline Fibras (g) & $5,46^{\mathrm{a}} \pm 1,22$ & $2,66^{\mathrm{b}}+1,15$ \\
Lipídios (g) & $4,94^{\mathrm{b}} \pm 1,26$ & $17,21^{\mathrm{a}}+1,36$ \\
\hline
\end{tabular}

*Médias seguidas de letra distinta diferem significativamente entre si pelo teste $\mathrm{T}(\mathrm{P}<0,05)$.

Segundo a Portaria no‥27, de 13/01/98, da Secretaria de Vigilância Sanitária do ministério da Saúde (BRASIL, 1998), são considerados fonte de fibras ou com aumentado teor de fibras os produtos prontos para o consumo que apresentarem no mínimo $3 \mathrm{~g}$ de fibras em 100g; portanto, pode-se considerar o biscoito recheado elaborado como fonte de fibras, principalmente quando comparado com o biscoito recheado industrializado.

KRÜGER et al. (2003) elaboraram dois tipos de biscoitos, tipo cookie e snack, utilizando para o enriquecimento proteico a caseína e o caseinato de sódio. Verificaram, após análise, que o biscoito tipo cookie apresentou teores mais elevados de fibra total que o do tipo snack, o que, segundo os pesquisadores, se deve provavelmente à aveia utilizada na formulação.

GUTKOSKI et al. (2007) também adicionaram aveia às barras de cereais visando ao desenvolvimento de barras de cereais com alto teor de fibras e igualmente obtiveram resultados satisfatórios.

Segundo SLAVIN (2004), esse cereal possui um alto teor de fibra alimentar, o que provavelmente contribuiu para o elevado teor de fibras encontrado nas barras de cereais elaboradas; contudo, optou-se por não utilizar aveia no biscoito recheado elaborado (BRE), pois, após serem realizados testes preliminares com adição desse cereal, verificou-se que os biscoitos ficaram com o sabor um pouco forte e, por se tratar de crianças, isso poderia interferir na aceitação.

Ainda em outro estudo realizado por PEREZ \& GERMANI (2007), no desenvolvimento de biscoito tipo salgado com farinha de berinjela, observou-se alto teor de fibras nos biscoitos elaborados, pois, segundo esses autores, a farinha de berinjela possui elevado teor de fibras, podendo ser classificados como produtos prontos para consumo com alto teor de fibras.

Em relação ao teor de lipídios (Tabela 1), o biscoito recheado elaborado (BRE) obteve 4,94g de lipídios em $100 \mathrm{~g}$, enquanto que o biscoito recheado industrializado (BRI) apresentou 17,21g de lipídios em $100 \mathrm{~g}$, ou seja, o biscoito recheado elaborado (BRE) apresentou menor teor de gordura do que a amostra industrializada, demonstrando diferença estatística significativa entre as amostras avaliadas.
Um estudo semelhante foi realizado por GAIDUS et al. (2007), os quais buscaram desenvolver biscoitos tipo waffer com reduzido teor de gordura trans. No estudo citado, os pesquisadores desenvolveram três diferentes formulações de recheios: uma contendo gordura hidrogenada $(\mathrm{GH})$, outra gordura hidrogenada interesterificada (GHI) e gordura de palma (GP). A análise sensorial sugeriu que a GHI e a GP são alternativas viáveis para a substituição da gordura hidrogenada, uma vez que, na maioria dos atributos, não houve diferença estatística entre os resultados.

Neste estudo, o BRE foi desenvolvido sem a adição de GH e GHI, para se verificar a viabilidade de se desenvolver um produto isento desse tipo de gordura. Em outro estudo realizado por CAPRILES \& ÁREAS (2005), a gordura vegetal hidrogenada foi substituída por óleo de canola para o desenvolvimento de salgadinhos com teores reduzidos de gordura saturada e ácidos graxos trans. Esses autores concluíram que é possível desenvolver um novo produto com alta aceitabilidade sensorial e com reduzidos teores de gordura saturada e isento de ácidos graxos trans.

Como pode ser observado na figura 1 , na avaliação geral da preferência, o BRE obteve 100\% de aceitação, enquanto que o BRI obteve $96 \%$ de aceitação. Além disso, verificou-se que não houve diferença estatística $(\mathrm{Pp}=2,83)$ entre o biscoito elaborado quando comparado com outra marca disponível no mercado e de consumo usual pelas crianças. Resultados similares foram observados por RODRIGUES et al. (2007), os quais analisaram sensorialmente formulações de biscoitos tipo cookie.

Em um estudo de FASOLIN et al. (2007), no qual foram produzidos biscoitos com substituição parcial de farinha de trigo por farinha de banana verde na proporção de 10, 20 e 30\% e biscoitos-padrão sem farinha de banana verde, verificou-se, por meio de teste de aceitação com 30 crianças, que o biscoito com $30 \%$ de farinha de banana verde foi o menos apreciado entre as crianças, diferindo significativamente dos biscoitos com $10 \%$ de farinha de banana verde do de $20 \%$ de farinha de banana verde e do padrão. A partir dos resultados desse estudo, pode-se inferir que as crianças fazem suas escolhas de acordo com o que realmente julgam ser o melhor.

Contudo, verificou-se que existem poucos trabalhos relacionados à análise sensorial com crianças e biscoitos, produto muito consumido por esse grupo. Dessa forma, há a necessidade de serem desenvolvidos mais estudos relacionados a esse assunto para que possam ser obtidos dados mais confiáveis. 


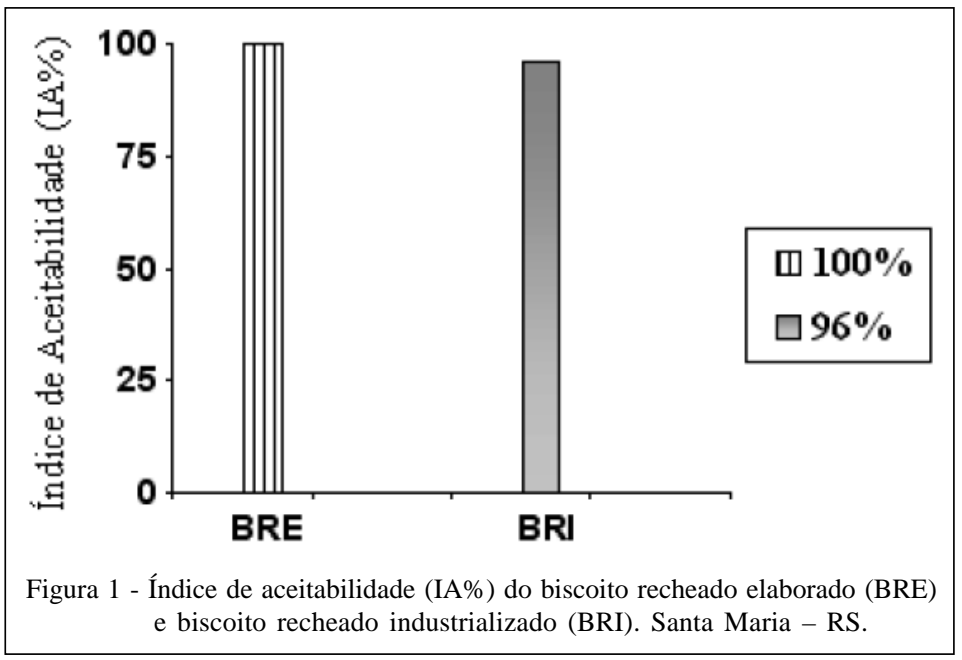

A elaboração de um biscoito recheado foi viável, pois este apresentou maior teor de fibras e menor teor de lipídios. O biscoito foi principalmente mais nutritivo se comparado ao biscoito recheado industrializado. Além disso, o produto demonstrou atributos sensoriais aceitáveis perante os escolares, tornando-se alternativa mais saudável na tecnologia de desenvolvimento de novos produtos.

\section{REFERÊNCIAS}

ASSOCIATION OF OFFICIAL ANALYTICAL CHEMISTS. Official Methods of Analysis. 16.ed. Washington: AOAC, 1995. p.1141,

BRASIL. Ministério da Saúde. Secretária de Vigilância Sanitária. Altera, e dá outras providências. Portaria n 27, de 13 de janeiro de 1998. Diário Oficial da União, Brasília, DF, 16 jan. 1998. Seção 1, p.1-3.

CAPRILES, V.D.; ARÊAS, J.A.G. Desenvolvimento de salgadinhos com teores reduzidos de gordura saturada e de ácidos graxos trans. Revista Ciência e Tecnologia de Alimentos, v.25, n.2,p.363-369,2005. Disponível em: <http:// www.scielo.br/ scielo.php?script=sci_arttext\&pid= S010120612005000200031>. Acesso em: 18 nov. 2009. doi: $10.1590 / \mathrm{S} 0101-20612005000200031$

FASOLIN, L.H. et al. Biscoitos produzidos com farinha de banana: avaliações química, física e sensorial. Revista Ciência e Tecnologia de Alimentos, v.27, n.3, p.524-529, 2007. Disponível em: <http://www.scielo.br/pdf/cta/v27n3/ a16v27n3.pdf $>$. Acesso em: 18 nov. 2009. doi: 10.1590/ S0101-20612007000300016.

GAIDUS, C.L. et al. Avaliação sensorial de biscoitos tipo wafer sabor chocolate com reduzido teor de gordura trans. Revista Nutrição Brasil, v.6, n.4, p.223-229, 2007.

GALLAGHER, E. et al. Use of response surface methodology to produce functional short dough biscuits. Journal of Food Engineering. v.56, n.2-3, p.269-271, 2003.

GUTKOSKI, L.C. et al. Desenvolvimento de barras de cereais à base de aveia com alto teor de fibra alimentar. Revista Ciência e Tecnologia de Alimentos, v.27, n.2, p.355363, 2007. Disponível em: <http:// www.scielo.br/scielo.php?pid=S0101$20612007000200025 \&$ script=sci abstract\&tlng=pt>. Acesso em: 18 nov. 2009. doi: 10.1590/S010120612007000200025.

KRÜGER, C.C.H. et al. Biscoitos tipo cookie e snack enriquecidos, respectivamente com caseína obtida por coagulação enzimática e caseinato de sódio. Revista Ciência e Tecnologia de Alimentos, v.23, n.1, p.8186, 2003. Disponível em: <http:// w w w. n u t r i c a o. u f p r. b r / biscoito_tipo_cookie.htm>. Acesso em: 18 nov. 2009. doi: 10.1590/S010120612003000100017.

LIMA, S.C.V.C. et al. Avaliação da dieta habitual de crianças e adolescentes com sobrepeso e obesidade. Revista de Nutrição, v.17, n.4, p.469-477, 2004.

MONTEIRO, A.G.R. Produção de biscoitos. São José do Rio Preto, UNESP, 1996. 56p. (Relatório de Estágio Supervisionado - apresentado ao departamento de engenharia e tecnologia de Alimentos).

ORMENESE, R.de C.S.C. et al. Perfil Sensorial e teste de consumidor de biscoito recheado sabor chocolate. Boletim Centro de Pesquisa de Processamento de Alimentos, v.19, n.2, p.277-300, 2001. Disponível em: <http://ojs.c3sl.ufpr.br/ ojs2/index.php/alimentos/article/view/1239>. Acesso em: 18 nov. 2009. doi: 10.1590/S0101-20612007000100033.

PEREZ, P.M.P.; GERMANI, R. Elaboração de biscoitos tipo salgado, com alto teor de fibra alimentar, utilizando farinha de berinjela. Revista Ciência e Tecnologia de Alimentos, v.27, n.1, p.186-192, 2007. Disponível em: <http:// www.scielo.br/scielo.php?script=sci arttext\&pid=S010120612007000100033>. Acesso em: 18 nov. 2009. doi: 10.1590/S0101-20612007000100033.

Farinha mista de trigo e berinjela: características físicas e químicas. Centro de Pesquisa e Processamento de Alimentos, v.22, n.1, p.15-24, 2004. Disponível em: <http:/ /ojs.c3sl.ufpr.br/ojs2/index.php/alimentos/rt/metadata/1176/0>. Acesso em: 18 maio de 2009. doi:10.1590/S010120612007000100029.

PITEIRA, M.F. et al. Extensional flow behaviour of natural fibre-filled dough and its relationship with structure and properties. Journal of Non-Newtonian Fluid Mechanics, v.137, n.1-3, p.72-80, 2006.

PUFFET, N. The use of linseed: managing constipation naturally. Journal Articles, v.18, n.6, p.10-13, 2004.

RODRIGUES, M. de A.A. et al. Desenvolvimento de formulações de biscoitos tipo cookie contendo café. Revista Ciência e Tecnologia de Alimentos, v.27, n.1, p.5-9, 2007. Disponível em: <http://www.scielo.br/scielo.php?pid=s010120612007000100029\&script=sci_arttext $>$. Acesso em: 18 nov. 2009. doi: 10.1590/S0101-20612007000100029

SLAVIN, J. Whole grains and human health. Nutrition Research Reviews, v.17, n.2, p.99-110, 2004. 